\title{
Assessing track records
}

SIR - Your correspondents Giovanni Motta and Neil B. Metcalfe (Nature 376, $720 ; 1995)$ criticize journal impact factors (mean citations per paper) as a means of evaluating scientific track records. Although impact factors may on their own provide a flawed assessment of an individual's performance, more sophisticated analysis can provide useful and objective information.

At the Unit for Policy Research in Science and Medicine (PRISM) at the Wellcome Trust, we have been undertaking experimental analyses to ascertain whether it is possible to improve on the simple use of impact factors when assessing publication performance. Three considerations have driven us: (1) the fact that performance is not distributed normally (neither in citations, nor other areas of human endeavour); (2) the search for appropriate benchmarks against which individuals can be compared; and (3) a belief that multiple indicators of performance will be more reliable than single measures.

An essential feature of our analysis is the definition of a cohort of comparison papers. In particular, we generate subfield-specific citation benchmarks that are appropriate for each applicant. We cannot rely on a subset of specialist journals, as many applicants work in a very narrow area and often publish their better papers in general journals. We therefore retrieve relevant papers by means of a complex title keyword filter, determined by consultation with scientists knowledgeable about the field. The comparison cohort should ideally comprise several hundred papers.

We then retrieve articles, notes and reviews by an applicant from the Science Citation Index and characterize each publication by the number of pages $(P)$, the number of authors $(A)$ and a journal weighting on a scale of 1 to 4 (the best) $(W)$. A consideration when a journal is being weighted is that the typical citation distribution is distinctly nongaussian, making the mean (as used for impact factors) an unreliable measure of central tendency (Fig. 1). We therefore employ categorical analysis, where journal weighting depends on how its citations relate to those of the cohort of comparison papers. We then calculate fractional outputs for each paper in the form of $P \mid A, W / A$ and $W P \mid A$.

We also determine the $C_{0-4}$ values (citations received over a five-year period) for each research paper on which the applicant is an author. These values are then compared with the distribution of $C_{0-4}$ values for the comparison cohort in order to determine in which quartile each article lies. An article in the top quartile is given a $C Q$ value of 4 , and an article in the bottom quartile is given a $C Q$ value of 1 . Papers in the top 10 per cent and top 5 per cent are given additional annotations.

The applicant's list of publications can also be compared with the track records of other leading scientists in the same scientific area. A useful indicator here is $W P / G$, where $G$ is the number of authors

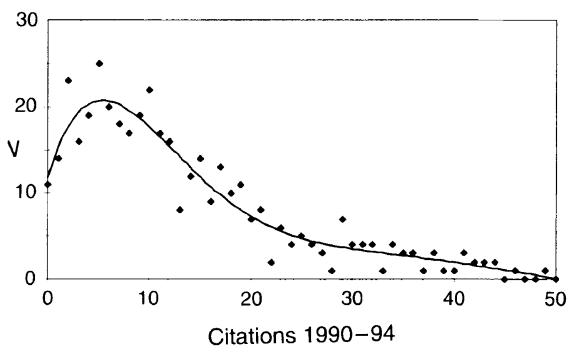

FIG. 1 The distribution of $C_{0-4}$ values (citations received by each paper over a five-year period) for the 424 papers published in 1990 in the journal Neuroscience: the mean is 16.4 but the median is 11 and the maximum is 340 .

in the group with whom the applicant has copublished. This measure shows scientists who either have large groups of their own or collaborate extensively with others, and may thus obtain credit for administrative rather than scientific brilliance.

We believe that our group of publication indicators can provide grants committees with useful input that they can then interpret in the light of their specialist knowledge in order to form an overall judgement on the scientific track record of applicants. All our analyses are based on the premise that publication lists are reasonable measures of track records, which is not necessarily true. Nevertheless, the publication lists of applicants continue to be referred to by grants committees when they are making awards. If inspection of publication records is to continue as part, but only a part, of the peer review process, the analysis must be carried out with the same degree of intellectual rigour that would be expected in the conduct of good scientific research. The alternative is to risk undue influence from subjective impressions, hearsay, anecdote and simple prejudice.

\section{Grant Lewison}

Joe Anderson

PRISM,

The Wellcome Trust,

210 Euston Road,

London NW1 2BE, UK

Julian Jack

University Laboratory of Physiology,

University of Oxford,

Parks Road,

Oxford OX1 3PT, UK

\section{Mind mechanisms}

SIR - Neuroscientists now study consciousness and will probably soon investigate the molecular biology of the mind. In parallel some argue that the mind houses mechanisms that enable people to develop multiple personalities, a sign of psychopathology that has increased dramatically recently ${ }^{1}$. There is no scientific foundation for this development ${ }^{1}$ and one wonders how it is possible now when molecular biology permeates clinical medicine.

It is unlikely that science can benefit from non-scientific developments. However, the reverse seems true. Thus, a representative of the non-scientific approach feels: "... on common ground with molecular biologists. Working with the multiple personality disorder patient, one has a sense of ... looking directly at its (the mind's) basic structure..." .

The stimulatory effect of growing scientific knowledge on non-scientific approaches to the questions of life is well known but has not been explained. Perhaps it reflects our ignorance about how one mind interacts with another, that is our limited understanding of interpersonal relations. Knowledge of these might solve some mental health problems, because the relationship between patient and mental health professional is distorted. In a remarkable study published 22 years ago, mental health professionals could not distinguish the mentally ill from those with no mental illness. By contrast, the mentally ill could ${ }^{2}$. Although this study was not followed up, distinguishing patient from pseudopatient may remain a problem because 75 per cent of the symptoms in the commonly used diagnostic manual of mental disorders are examples of normal behaviour ${ }^{3}$.

Understanding interpersonal relations is the 'big question' now facing biologists. Neuroscience and molecular biology may help in answering the question, but new methods will be needed, suggests a molecular biologist ${ }^{4}$.

\section{Per Södersten}

Karolinska Institute,

Department of Clinical

Neuroscience,

Novum, S-141 57 Huddinge,

Sweden

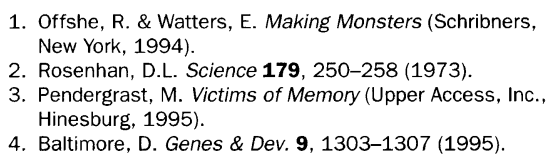

Correction: Eradicating nuclear weapons The last sentence of the letter by Jan $\mathrm{H}$. J. Oelering (Nature 377, 10; 1995) should have read: "So rather than a few months after Hiroshima, publication [of the Smyth report] took place a few days after Hiroshima". 